\title{
dentistry 2005
}
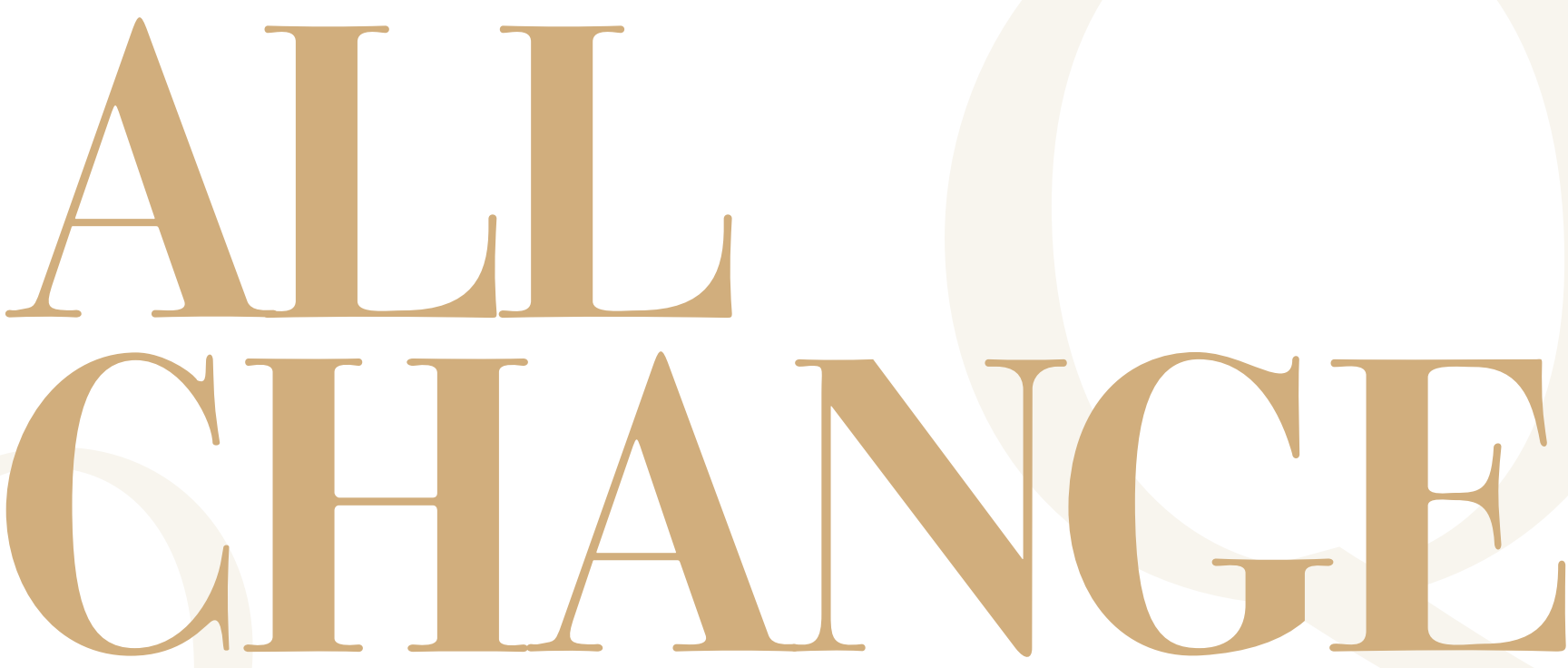

The biggest reforms to NHS dentistry in England in Wales since its inception are due to be implemented in October 2005. What does all this mean for members of the wider dental team? Andrew Hoy answers some commonly asked questions.

\section{'The Department of Health hopes that the reforms} might encourage more of a focus

on team working within a practice?

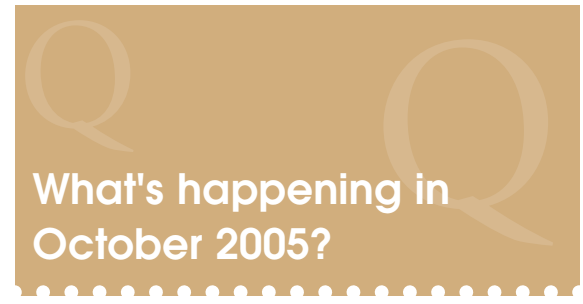

There are two main changes, the first of which is a move of commissioning of General Dental Service (GDS) services at PCT level. The second change is that there will be a move away from item-of-service dental payments to regular monthly payments to dentists. These regular monthly payments will generally be based on the historic NHS income of a practice.

For the salaried services, the current functions of Salaried Primary Dental Care will continue under PCT-provided primary dental services, and will operate alongside the GDS. Such services will include the provision of treatment to patients who might not otherwise seek treatment, for example patients with special needs.
Other processes going on at the same time, include a review of the patient charges system, a revision of new dental recall guidelines by the National Institute of Clinical Excellence (NICE), and work on dental Information Technology systems within the NHS.

\section{Why are they happening?}

The government decided to push forward with these changes, in the wake of the publication of the Options for Change report in August 2002. The Government thinks that transferring dental commissioning in PCTs will offer some exciting opportunities for co-ordinated and consistent delivery of dental services, and opportunities for services tailored to local needs. The Government also 
dentistry 2005:

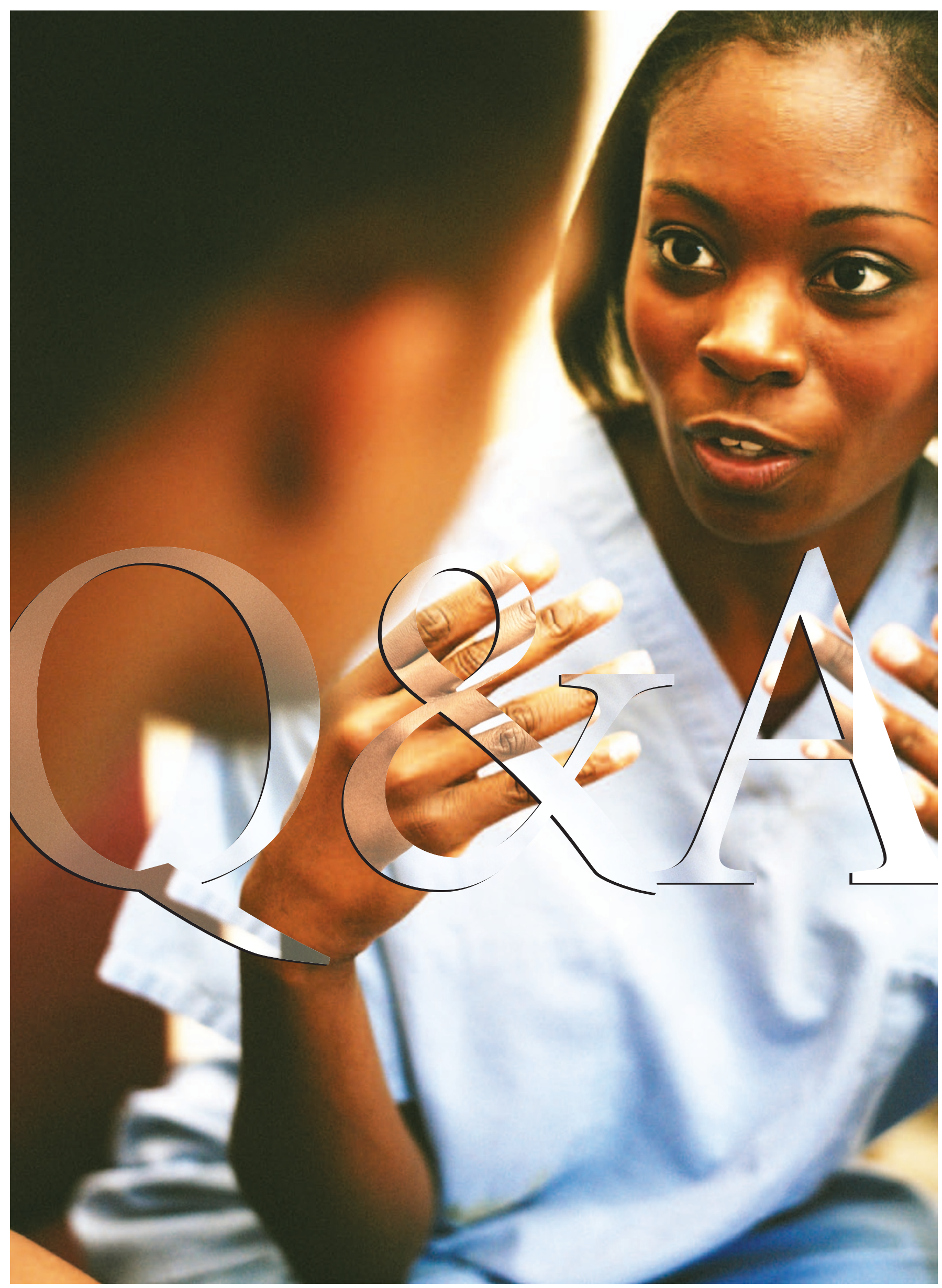

autumn $04 \mathbf{2 3}$ vital 
hopes it will also allow dentists and their staff to get off the current 'treadmill' of dental remuneration.

\section{How will patients react to the changes?}

Patients may have a number of questions relating to the reforms, including questions about the right to access NHS dental treatment and register as a patient, changes to patient charges, and the changes to dental recall intervals. It may be receptionists who field most of these questions.

Because some of these issues are still being discussed with the Government as part of the development of the new base contract needed to implement the reforms, it will be some time before definitive answers can been given to many of these questions. The profession (through the BDA) is continuing to press for these details to be finalised and made available to people in dental practices.

In the meantime though, practice staff should obviously maintain an interest in the reforms, and try to become well versed in the details of the changes as they become available. Vital will continue to update you as the change approaches.

\section{How should staff respond to some of these questions?}

Changes to dental recall guidelines will come in at the same time of the reforms and may mean that some patients with good oral health will be able to visit the dentist less often for a check-up. Patients in these cases may feel that they are endangering their teeth, as they compare their new recall interval to the relatively frequent check-ups (sixmonthly) that exist currently. Patients in this situation may need to be reassured that their level of oral health means that a shorter recall internal would not be justified.

What is happening with the new patient charges regime is also still unknown, but it is expected to be a clearer and more transpar- ent system. While this may make it easier to explain to patients, it is possible that the prices of some categories of treatment could rise, and the prices of other categories of treatment could fall. Some patients may be annoyed by any price rises. It may be useful to point out to them that there is no relationship between the amount of additional money collected as a result of any charge increases and the amount of money paid to the practice by the Government.

\section{How will technicians be afifected?}

Some dental technicians have expressed a fear that there will be less lab work under the new contract, although there have been wildly varying estimates of how much this reduction will be.

It may be that there will be a small reduction, or in fact no reduction at all. The position of the Department of Health is that while there are likely to be changes in the way that dentists use dental labs under the new arrangements, results from PDS pilot sites suggests a drop of only $2 \%$ in lab work ${ }^{1}$. Dentists will also be monitored with respect to the adequacy of their prescribing if their treatment that involved lab work fell below a certain level.

Possibly there could even be an increase in lab work in the near future. The Government announced in July 2004 that it intended to increase the NHS dental workforce by 1000 additional dentists by October 2005. The presence of more dentists may contribute to a national increase in the demand for lab work that may offset any reduction that occurs.

\section{Will hygenists/therapists change their work patterns?}

In the recently released workforce review, the Department of Health noted that the impact of the reforms would be complex, but engaged in some speculation about what might happen. The Department of Health hopes that the reforms might encourage more of a focus on team working within a practice, with some PCDs taking over some types of work from dentists, enabling more effective deployment of dentists' specialist skills. Possibly this may happen, although some of this teamwork is already happening as a result of extensions in the last couple of years to the range of duties dental therapists and dental hygienists may carry out.

\section{What are Personal Dental Services (PDS) contracts?}

PDS is another type of contract that a practice or a dentist can use to deliver dental services, as an alternative to GDS-type arrangements. In recent months, the Government has accelerated the rate at which PDS arrangements are being implemented across the country, and PCDs may find that their practice moves to one of these contracts.

The PDS process is separate from the 2005 reforms, but does have two key connections. Firstly, the contracts are very similar to the block payment approach that is coming in for all other practices in 2005. Secondly, some dentists are keen to move to the new method of remuneration prior to the implementation of the reforms, and PDS offers them the opportunity of doing so.

\section{Where do I go for more information?}

You can access up to date information from the BDA's website, www.bda.org. From the front page, click on 'Advice and Resources' at the bottom, and then click on 'Update 2005'.

1. Barry Cockcroft, Dental Laboratory, June 2004.

This issue's 'Your View' is devoted to the Dentistry 2005 changes. To see how team members feel the changes will affect them, turn to page 15. 